\title{
Experimental and Computational Solid Form Landscape of a Pharmaceutical Molecule
}

\author{
Rajni M. Bhardwaj, \\ Small Molecule Design and Development, Eli Lilly \& Company, Indianapolis, IN 46285, USA \\ bhardwaj_rajni@lilly.com
}

\begin{abstract}
The solid form screening of a pharmaceutical compound " $\mathrm{A}$ " produced many solvates, prompting an extensive investigation into possible risks to the development of the favored monohydrate form. Inspired by crystal structure prediction, the search for neat polymorphs was expanded to an unusual range of experiments, including crystallization of the melt under pressure, to work around solvate formation and the thermal instability of the molecule. This presentation will highlight how state-of-the-art experimental techniques, characterization methods can be combined with emerging computational modelling techniques to produce a complete solid-state structure landscape (various polymorphs and solvates). Also, how this unprecedented access to crystallographic information has paved the way for a detailed analysis of the structures in relation to their properties, as well as an assessment of the completeness of the experimental search for neat polymorphs by comparison to structures on computed crystal energy landscapes will be discussed.
\end{abstract}

